\title{
Microbial population heterogeneity versus bioreactor heterogeneity: evaluation of Redox Sensor Green as an exogenous metabolic biosensor
}

Baert, Jonathan; Delepierre, Anissa; Telek, Samuel; Fickers, Patrick; Toye, Dominique; Delamotte, Anne; Lara, Alvaro R.; Jaén, Karim E.; Gosset, Guillermo; Jensen, Peter Ruhdal

Total number of authors:

11

Published in:

Engineering in Life Sciences (Online)

Link to article, DOI:

10.1002/elsc.201500149

Publication date:

2016

Document Version

Peer reviewed version

Link back to DTU Orbit

Citation (APA):

Baert, J., Delepierre, A., Telek, S., Fickers, P., Toye, D., Delamotte, A., Lara, A. R., Jaén, K. E., Gosset, G., Jensen, P. R., \& Delvigne, F. (2016). Microbial population heterogeneity versus bioreactor heterogeneity: evaluation of Redox Sensor Green as an exogenous metabolic biosensor. Engineering in Life Sciences (Online), 16(7), 643-651. https://doi.org/10.1002/elsc.201500149

\section{General rights}

Copyright and moral rights for the publications made accessible in the public portal are retained by the authors and/or other copyright owners and it is a condition of accessing publications that users recognise and abide by the legal requirements associated with these rights.

- Users may download and print one copy of any publication from the public portal for the purpose of private study or research.

- You may not further distribute the material or use it for any profit-making activity or commercial gain

- You may freely distribute the URL identifying the publication in the public portal 
Microbial population heterogeneity versus bioreactor heterogeneity: evaluation of Redox Sensor Green as an exogenous metabolic biosensor

Jonathan Baert ${ }^{1}$, Anissa Delepierre ${ }^{1}$, Samuel Telek ${ }^{1}$, Patrick Fickers ${ }^{1}$, Dominique Toye ${ }^{2}$, Anne Delamotte ${ }^{2}$, Alvaro R. Lara ${ }^{3}$, Karim E. Jaén ${ }^{3}$, Guillermo Gosset ${ }^{4}$, Peter R. Jensen ${ }^{5}$, Frank Delvigne1,*

1 University of Liège, Gembloux Agro-Bio Tech, Microbial Processes and Interactions (MiPI), Passage des Déportés, 5030 Gembloux, Belgium

2 University of Liège, Department of Chemical Engineering - Product, Environment and Processes (PEPs), Allée du 6 Aout, 11(bât B6c), 4000 Liège, Belgium

3 Departamento de Procesos y Tecnología, Universidad Autónoma Metropolitana-Cuajimalpa, Vasco de Quiroga 4871, Col. Santa Fe, Cuajimalpa, C. P. 05348, México, D. F.

${ }^{4}$ Instituto de Biotecnología, Universidad Nacional Autónoma de México, Apdo. Postal 510-3, Cuernavaca, Morelos 62210, México

5 National Food Institute, Technical University of Denmark (DTU), Building 221, room 140, DK2800 Lyngby, Denmark

- $\quad$ Corresponding author : F.Delvigne@ulg.ac.be

Practical application

It is known that isogenic microbial population is able to display phenotypic diversification upon environmental changes. In this study, we developed a high throughput methodology based on the use of flow cytometry and the preliminary staining of the cells with Redox Sensor Green (RSG). We demonstrated that RSG is related to the activity of the electron transport chain and

Received: 10 22, 2015; Revised: 04 28, 2016; Accepted: 06 15, 2016

This article has been accepted for publication and undergone full peer review but has not been through the copyediting, typesetting, pagination and proofreading process, which may lead to differences between this version and the Version of Record. Please cite this article as doi: 10.1002/elsc.201500149.

This article is protected by copyright. All rights reserved. 
can be used as an efficient tracker of metabolic activity at the single cell level in different bioreactor configurations. We showed that RSG can be efficiently used to detect carbon limitation and dissolved oxygen limitation in bioreactor. More specifically, our methodology was used in order to assess a potential relationship between bioreactor heterogeneity and microbial population heterogeneity. Unexpectedly, no such correlation was found based on scale-down analysis.

Abstract

Microbial heterogeneity in metabolic performances has attracted a lot of attention, considering its potential impact on industrial bioprocesses. However, little is known about the impact of extracellular perturbations (i.e. bioreactor heterogeneity) on cell-to-cell variability in metabolic performances (i.e. microbial population heterogeneity). In this work, we have evaluated the relevance of Redox Sensor Green (RSG) as an exogenous biosensor of metabolic activity at the single cell level. RSG signal is proportional to the activity of the electron transport chain and its signal is strongly affected by metabolic burden, availability of electron final acceptor and side metabolisms (i.e. overflow and mixed acid fermentation). RSG can also be used for the estimation of the impact of scale-down conditions on microbial metabolic robustness. The relationship linking averaged RSG activity and its cell-to-cell variability (noise) has been highlighted but seems unaffected by environmental perturbations.

Keywords: single cell, scale-down, biological noise, flow cytometry, phenotypic heterogeneity

Introduction

Bioreactor scale-up drives the appearance of gradients that are able to trigger significant physiological reactions at the level of the microbial population [1][2]. Indeed, microbial cells are exposed to different micro-environmental conditions (i.e., $\mathrm{pH}$, dissolved oxygen, nutrients,...) inside heterogeneous bioreactor varying in amplitude with time and the specific location inside the bioreactor [3][4]. An important fundamental question at this level is to know whether environmental fluctuations typically found in large-scale bioreactor are able to drive phenotypic heterogeneity at the level of the microbial population [5]. In fact, cell-to-cell phenotypic variability is driven by two components, i.e. an intrinsic source of noise resulting from stochasticity of reaction between molecular components, and an extrinsic source of noise resulting from variability in copy number of these molecular components [6] [7]. This last component is notably influenced by the history of micro-environmental conditions met at the single cell level and is thus strongly linked with bioreactor heterogeneities. The intrinsic

This article is protected by copyright. All rights reserved. 
component of noise is actually relatively well defined, with several modeling approaches, relying on stochastic equations for gene expression and protein synthesis [6]. However, little is known about the real influence of environmental factors on the extrinsic component of noise, and additional experiments are thus needed at this level [8]. For the remaining of this work, the terms stochasticity, noise and phenotypic heterogeneity will be used in order to express cell-tocell differences among the same population of microbes. These terms can be used in order to express the same phenomenon, but at different stages, i.e. stochasticity in biochemical reactions induces the appearance of noise, which in turn is able to drive phenotypic heterogeneity among the microbial population. Besides its impact on gene expression and protein synthesis, phenotypic variability in metabolic performances recently attracted a lot of attention considering its potential impact on bioprocesses [9]. It has been notably shown that segregation in single cell metabolic activities occurs upon shift in nutrient conditions, and more precisely upon shift from glycolytic to gluconeogenic carbon sources [10][11][12]. Since diauxic shift is likely to occur in standard process conditions (e.g., acetate is produced by E. coli by overflow metabolism and can be consumed upon glucose limitation in fed-batch bioreactor), the occurrence of metabolic variability in heterogeneous bioreactors must be assessed. Different single cell technologies are available for this purpose, including microfluidics cultivation devices [13] and flow cytometry [14]. These two techniques have pros and cons, i.e. ease of use and automation for flow cytometry and spatial and time resolution of analysis in the case of single cell microfluidic devices [9]. In the context of this work, effect of fluctuating environmental conditions was estimated in scale-down bioreactors, leading to the choice of flow cytometry as a single cell method. Another important technical point is related to the design of efficient biosensing technologies for detecting the occurrence of microbial phenotypic heterogeneity [15][16]. Genetically-encoded biosensors based on the Green Fluorescent Proteins (GFP) have been widely used for the detection of microbial stress [17], the production of recombinant proteins [18] or the metabolic specialization of microbial cells [19]. However, the dynamic range of most of the GFP variants is not suitable for the detection of fast evolving processes [20][21], such as metabolic related ones. An alternative to genetically-encoded biosensors is to use exogenous biomarkers [22]. In the context of this work, cell-to-cell variability was estimated based on the use of an exogenous sensor, i.e. Redox Sensor Green (RSG). RSG has been used as a vitality indicator so far, but we find out that its use would be extended to the detection of metabolic activities at a single cell level. Indeed, RSG is reduced by the intracellular reductases involved in the aerobic metabolism involved in the electron transport chain [23], leading to the release of a green fluorescent compound that can be easily detected by flow cytometry. RSG could thus be considered as a key indicator of the metabolic state of the microbial cells in different bioprocessing conditions. Dynamic range of RSG will thus be first determined in standard well-mixed batch reactor, on the basis of wild-type strain and mutants showing either improved or decreased electron transport efficiencies. Upon validation, RSG will be used in order to decipher the occurrence of phenotypic heterogeneity in function of the external perturbations found in different scale-down reactor set-ups. For this purpose, noise in RSG activity will be used as a proxy for expressing cell-to-cell differences in metabolic activity.

Material and methods

This article is protected by copyright. All rights reserved. 
1. Strains and medium

All strains were maintained at $-80^{\circ} \mathrm{C}$ in working seeds vials $(2 \mathrm{~mL})$ in solution with $40 \%$ of glycerol. Precultures and cultures were performed based on a defined mineral salt medium containing (in g/L): $\mathrm{K}_{2} \mathrm{HPO}_{4}$ 14.6, $\mathrm{NaH}_{2} \mathrm{PO}_{4} \cdot 2 \mathrm{H}_{2} \mathrm{O} 3.6, \mathrm{Na}_{2} \mathrm{SO}_{4} 2,\left(\mathrm{NH}_{4}\right)_{2} \mathrm{SO}_{4} 2.47, \mathrm{NH}_{4} \mathrm{Cl} 0.5$, $\left(\mathrm{NH}_{4}\right)_{2}$-H-citrate 1, glucose monohydrate 10 (excepted for the scale-down experiments, where glucose monohydrate concentration was increased to $20 \mathrm{~g} / \mathrm{L}$ ), thiamine 0.01 . Thiamin is sterilized by filtration $(0.2 \mu \mathrm{m})$. The medium was supplemented with $3 \mathrm{~mL} / \mathrm{L}$ of trace elements solution, $3 \mathrm{~mL} / \mathrm{L}$ of a $\mathrm{FeCl}_{3} \cdot 6 \mathrm{H}_{2} \mathrm{O}$ solution $(16.7 \mathrm{~g} / \mathrm{L}), 3 \mathrm{~mL} / \mathrm{L}$ of an EDTA solution $(20.1 \mathrm{~g} / \mathrm{L})$ and $2 \mathrm{~mL} / \mathrm{L}$ of a $\mathrm{MgSO}_{4}$ solution $\left(120 \mathrm{~g} / \mathrm{L}\right.$ ). The trace elements solution contains (in g/L): $\mathrm{CoCl}_{2} \cdot \mathrm{H}_{2} \mathrm{O}$ $0.74, \mathrm{ZnSO}_{4} \cdot 7 \mathrm{H}_{2} \mathrm{O} 0.18, \mathrm{MnSO}_{4} \cdot \mathrm{H}_{2} \mathrm{O} 0.1, \mathrm{CuSO}_{4} \cdot 5 \mathrm{H}_{2} \mathrm{O}, \mathrm{CoSO}_{4} \cdot 7 \mathrm{H}_{2} \mathrm{O}$. In addition with the wild-type Escherichia coli W3110, two genetically engineered strains were considered further for the analysis of the RSG activity. The first one was E. coli BL21 expressing VHb. The chromosomal integration of the Vitreoscilla stercoraria hemoglobin gene ( $\mathrm{vg} b$ ) in Escherichia coli BL21 (DE3) was performed following a protocol previsouly developed [24]. The $v g b$ gene was amplified by PCR from plasmid pNKD1 [25] (donated by Prof. Benjamin Stark, Illinois Institute of Technology, USA). The PCR product (463 bp) was cloned into pCRII-Blunt-TOPO and subcloned in pLoxGentrc between the NcoI and HindIII sites to yield pLoxGentrc- $v g b$. In such construction, the start codon of the $v g b$ gene is located downstream the trc promoter. The DNA region containing the trc promoter, $v g b$ and gentamicin-resistance genes flanked by Lox sequences was amplified by PCR from the pLoxGentrc- $v g b$ plasmid. The resulting product, that contains $50 \mathrm{nt}$ regions homologous to the genes lacI and lacZ, was used to transform Escherichia coli BL21 (DE3) bearing the plasmid was pKD46, in which the Red system proteins were already induced with arabinose. This way, the PCR product was integrated into the chromosome by homologous recombination between lacI and lacZ. The integration was confirmed by resistance to Gentamicin, by PCR amplification and subsequent sequencing of the PCR product.

The second strain was designed in order to investigate the effect of lowering the availability of ATP. E. coli K12 MG1655 was used as the strain background for F1-ATPase expression. The strain was transformed with a plasmid pCP34::atpAGD (high ATPase activity) [26]. Briefly, the atpAGD operon from E. coli, encoding the soluble F1 part of the F1F0-ATPase ( $\alpha, \beta$ and $\gamma$ subunits) has been inserted into the vector pAK80 under transcriptional control of a series of synthetic promoters.

2. Bioreactor operating conditions and scale-down set-ups

Before each bioreactor cultivation experiment, a precultivation step was performed in $100 \mathrm{~mL}$ based on the cultivation medium previously described. Precultivation was performed during $15 \mathrm{~h}$ in baffled shake flask at $37^{\circ} \mathrm{C}$ in orbital shaking incubator (Sanyo IOX402.XX1.C) at 130

This article is protected by copyright. All rights reserved. 
rounds per minute. For the batch experiments, cultures were performed in a Dasgip minibioreactor platform (DASGIP DASbox Reactor SR02500DLS). This platform allowed the parallel cultivation of four microbial strains. Except for the scale-down set-ups, these mini-bioreactors were filled with $200 \mathrm{~mL}$ of the defined medium described previously. All the bioreactors were inoculated with $10 \mathrm{ml}$ of cellular suspension provided by the precultivation step in order to reach an intitial optical density ranging between 0.4 and 0.5 . Mechanical stirring was provided by two Rushton turbines with 6 blades at an agitation rate of $1000 \mathrm{~min}^{-1}$. Air flow rate was maintained at $100 \mathrm{~mL} / \mathrm{min}$ and the $\mathrm{pH}$ was kept at 7.0 during the whole culture. For the scaledown experiments, $\mathrm{pH}$, temperature and airflow rate remain unchanged except that the stirring rate was increased to $1300 \mathrm{rpm}$ in order to avoid oxygen limitation. Furthermore, two different scale-down approaches were implemented for generating dissolved oxygen fluctuations. The first scale-down system consisted of a single stirred tank bioreactor operated in batch mode in which stirring was modulated according to an ON/OFF profile with duration of 1.25 and 1.80 minutes respectively. The second scale-down system was based on two interconnected stirred tank bioreactors operated in batch mode. The first bioreactor was fully supplied in oxygen and filled with $220 \mathrm{ml}$ of medium, whereas the second bioreactor was unaerated, stirred at $40 \mathrm{rpm}$ for ensuring homogeneity in the vessel, and filled with $150 \mathrm{ml}$ of medium. The continuous recirculation of the microbial cells between the two stirred tank reactors was performed with two external peristaltic pump. The first pump operated at a flow rate of $120 \mathrm{ml} / \mathrm{min}$ and the second pump operated at a flow rate of $200 \mathrm{ml} / \mathrm{min}$ but the sampling rod depth ensures that the volume in the unaerated bioreactor remains constant at $150 \mathrm{ml}$. By this way, the average residence time spent by the microbial cells under dissolved oxygen limitation was comparable between the two SRD set-ups (results not shown).

3. Analytical techniques

Cellular density was measured by spectrophotometry at $600 \mathrm{~nm}$ using a Genesys $10 \mathrm{~S}$ UV-Vis spectrophotometer (Thermo Scientific, Waltham, USA). Samples were previously diluted with phosphate buffer in order to obtain an absorbance ranging between 0.2 and 0.8. Glucose and acetate concentrations in the culture medium were determined using an Agilent 1200 series high performance liquid chromatograph (HPLC, Agilent Technologies) equipped with a Aminex HPX-87H column (Bio-RAD laboratories, Hercules, USA) preceded by a Supelguard H precolumn (Sigma-Aldrich, Saint-Louis, USA). The column were kept at a temperature of $40{ }^{\circ} \mathrm{C}$, and the isocratic mobile phase was $0.008 \mathrm{~N} \mathrm{H}_{2} \mathrm{SO}_{4}$ (in ultrapure, "milliQ", water), at a flow rate of $0.6 \mathrm{ml}$ min-1. A refractive index signal detector, kept at $40{ }^{\circ} \mathrm{C}$, was used. This analysis took $40 \mathrm{~min}$ at a maximum pressure of 70 bar. Prior analysis, samples were filtered with $25 \mathrm{~mm}$ CA Syringe Filters on a $0,22 \mu \mathrm{m}$ filter (ROCC, Sart-Eustache, Belgium) and supplemented with sodium azide at a final concentration of $0.05 \%$. Samples were stored at $10^{\circ} \mathrm{C}$ before analysis.

This article is protected by copyright. All rights reserved. 


\section{4. $\quad$ Flow cytometry and RSG staining}

RSG normally enters cells and is reduced by reductase enzymes related to cellular respiration compris in the little is known about the time required for the RSG to diffuse inside the cells and to be reduced. In order to determine the contact time required in order to obtain a stable signal by flow cytometry, real time flow cytometry has been used to characterize the kinetics of RSG reduction. For this purpose, samples taken at different growth stages during a batch culture of $E$. coli W3110 were analyzed. For all the cases, the time required in order to obtain a stable signal was around $24 \pm 1$ minutes (Supplementary file S1). Based on these elements, cells were systematically stained by adding $1 \mu \mathrm{L}$ Redox Sensor Green (Invitrogen, UK) in $1 \mathrm{ml}$ of cellular suspension and incubated during $25 \mathrm{~min}$ at $20^{\circ} \mathrm{C}$. Prior to this step, the cellular suspension was diluted with phosphate buffer saline (PBS pH $=7$ ) for adjusting the cell density between 500 and 2500 events/ $\mu \mathrm{L}$ during flow cytometry analysis. RSG's fluorescence was measured on the FL1 channel of a C6 Accury Flow Cytometer (BD Biosciences, NJ USA). Each analysis was performed by analyzing 40,000 events (forward scatter (FSC) threshold $>80,000$; sheat fluid flow rate set to medium). The raw data were then extracted as .fcs files and loaded into MatLab by using the readfsc function (by L. Balkay, University of Debrecen, Hungary, available on MatLab central file sharing). The FL1 channel (green fluorescence) was processed in order to compute the median, the mean and the standard deviation of RSG intensity at the level of the microbial population.

Results and discussion

1. Evolution of RSG staining during batch bioreactor cultivation

As stated before, RSG has been used only in a few studies as a viability indicator, and its relevance as metabolic marker has not been fully assessed [23]. In order to assess the efficiency of RSG staining as an indicator of metabolic activity in process conditions, a first set of batch bioreactors of E. coli W3110 have been carried out (Figure 1). The level of fluorescence attributed to RSG reduction increases during the main growth phase (Figure 1A). During this phase, glucose consumption is accompanied with acetate excretion by overflow metabolism (Figure 1B). When both substrates (i.e. glucose and acetate) are depleted, RSG signal drops rapidly and becomes very low when stationary phase of growth is reached. RSG signal seems thus to follow quite well the main metabolic transition during a batch culture of E.coli W3110 and could thus be used as an efficient metabolic tracer. Furthermore, it is interesting to notice that the level of RSG fluorescence is continuously increasing over time even after reaching the maximum growth rate. Indeed, RSG signal can only be correlated with the activity of the aerobic metabolic pathways. The increase of the fluorescence signal before the substrate depletion suggests a continuous increase of the electron transport capacity of the cells. On

This article is protected by copyright. All rights reserved. 
the other hand, the relative part of anaerobic metabolic pathways should decrease as a consequence. Therefore, it can be assumed that RSG analysis gives a relative insight on the carbon flux metabolized through the aerobic pathway in comparison with the carbon flux metabolized through fermentative pathway. It is important to point out that this first experiment has been carried out without any dissolved oxygen limitation. In the next section, the effect of dissolved oxygen limitation, as well as improved respiratory capacity and intrinsic metabolic burden, will be investigated.

\section{Effect of enhanced respiratory capacity and metabolic burden on RSG activity}

In order to further investigate the effect of external and internal factors on RSG signal, additional experiments involving dissolved oxygen limitation and mutants strains of $E$. coli have been carried out. In a first step, impact of dissolved oxygen limitation on E. coli W3110 and $E$. coli BL21 expressing the Vitreoscilla hemoglobin (VHb) was estimated (Figure 2). It can be observed that E. coli W3110 rapidly drops at the level of the RSG signal when aeration is turned off during a batch culture (Figure 2A). This observation is in accordance with the molecular mechanisms behind the generation of fluorescent signal from RSG. Indeed, when the final electron acceptor (oxygen) is depleted, activity of aerobic metabolic pathway the electron transport chain is arrested and RSG can no longer be reduced. On the opposite, E. coli BL21 expressing $\mathrm{VHb}$ is able to maintain a strong fluorescent signal upon oxygen depletion (Figure 2B). As stated previously, fluorescence emission of RSG is linked with the intracellular reductase activity, but a little is known about the metabolic role of these reductases and the biomolecular mechanism involved in the RSG response.

The expression vector was previously designed in order to increase $\mathrm{VHb}$ synthesis under fully aerobic conditions. It was shown that, in this case, overflow metabolism was reduced and plasmid DNA yield was increased [27][28]. VHb facilitates oxygen uptake from the media and delivers it to the cytochromes more efficiently, thus enhancing the electron transport chain activity. This mechanism tends to increase the robustness of the strain in front of dissolved oxygen fluctuations. But in fully anaerobic condition, $\mathrm{VHb}$ protein cannot improve the cellular aerobic metabolic activity. Oxygen starvations are likely to occur, at least transiently, in largescale bioreactors and are known to induce strong modifications at the level of the cells physiology [29], and this interesting feature can be detected by analyzing the RSG signal. Nevertheless, the RSG response towards the VHb-strain in both aerobic and anaerobic conditions were very helpful for deepening this issue. Indeed, as previously stated, the VHb protein plays a major role at the level on the electron transport chain activity in aerobic condition [28]. The RSG fluorescence for the VHb-strain in aerobic conditions is strongly increased in comparison with the W3110 strain. But a fluorescence signal remaining high even in anaerobic conditions was not expected. This observation suggest that the VHb protein plays a major role in the RSG fluorescence emission. Therefore, for the VHb-strain, the RSG response cannot be correlated to metabolic activity. Furthermore, previous studies stated that VHb protein itself harbors a terminal oxidase function [30], allowing the cellular growth on aerobic

This article is protected by copyright. All rights reserved. 
substrate of mutant lacking terminal oxidase [25]. Similarity between the oxidoreductive characteristics between the $\mathrm{VHb}$ protein and the terminal oxidase complex suggests that, apart for the VHb-strain, the cytochrome complex is tightly implicated in the reaction required for the RSG fluorescence emission. At this stage we are able to propose a reactional redox mechanism closely linked with the electron transport chain activity which explaining how the RSG becomes fluorescent. We suggest that only the RSG's reduced form emitted green fluorescence and its intensity is proportional with its reduction state. Furthermore the cytochrome complex seems plays a central role in this reduction reaction by transferring electron, initially provide by NADH reduction, to the RSG molecule. The greater is the electron flux through the electron transport chain, the higher will be the reduced state of the RSG molecule and the higher will be the fluorescence signal.

As a negative control, an E. coli strain expressing the F1-ATPase has been cultivated in batch mode and stained with RSG at different time intervals (Figure 3). It can be observed that this strain exhibits a very low RSG signal during the whole culture (Figure 3A). Indeed, expression of F1-ATPase is know to reduce the intracellular ATP concentration and increases significantly the glycolytic flux [26][31]. Consequently, the excess of carbon flux through the aerobic metabolic pathways is diverted towards the fermentative metabolism, as observed in our experimental conditions by the excretion of a large amount of acetate well known to be an overflow metabolite (Figure 3B). In this case, it seems that a major part of the carbon flux is diverted to the acetate pathway, leading to a significant reduction of the aerobic metabolism activity which induced a reduction of the RSG fluorescence signal.

We have thus shown that artificially modifying the intracellular state of the cell leads respectively to an increase in RSG activity (in the case of VhB expression) or to a decrease of this signal (i.e., in the case of the expression of the F1-ATPase). Since RSG signal can also be used for monitoring extracellular parameters, i.e. either about the carbon source or the electron final acceptor availability, it should be interesting to determine whether fluctuations in environmental conditions are able to drive cell to cell heterogeneity in metabolic activity. This question will be addressed in the next section.

Cell-to-cell heterogeneity in function of bioreactor heterogeneity

Phenotypic heterogeneity can be related to biological noise. This noise is typically measured on the basis of flow cytometry analysis of fluorescent reporter strains [32]. Results are typically represented as frequency distribution of fluorescence abundance among the microbial population. Noise can be calculated by taking the ratio of the standard deviation to the mean of these distributions (i.e., this ratio corresponding to the coefficient of variation CV) [33][34]. This methodology has been further extended in this work by considering frequency distributions corresponding to the RSG fluorescence. The question that has to be addressed here is to know whether biological noise is enhanced or not by environmental noise generated in bioreactors, and more precisely noise related to dissolved oxygen fluctuations. Indeed, in this case,

This article is protected by copyright. All rights reserved. 
environmental fluctuations have an impact at the level of the extrinsic component of biological noise, leading to a potential increase of phenotypic heterogeneity.

As an attempt to make the link between biological noise and bioreactor heterogeneity, comparative scale-down experiments were further conducted to assess the reliability of RSG biosensors to report the effect of fluctuation of dissolved oxygen availability for E. coli W3110 grown on glucose base media (Figure 4). The first culture was carried out in a standard stirred tank bioreactor without dissolved oxygen limitation. Two additional tests were carried out by considering two scale-down reactor (SDR) set-ups based on different principle for the induction of dissolved oxygen fluctuations. In the single-compartment SDR, dissolved oxygen level was varying based on the modulation of the stirrer speed at a given frequency. In the twocompartment SDR, recirculation of the microbial cell between two stirred tank reactors with different oxygen transfer rates was considered. It is important to point out that the average residence between the two compartments of this set-up match approximately the frequencies of exposure to dissolved oxygen limitation in the single-compartment SDR. Since these two set-ups are widely used in the literature [2], they can be considered as a good basis for estimating the impact of bioreactor heterogeneity on biological noise. Also, the mechanisms of cell exposure to dissolved oxygen limitation are fundamentally different between the two set-ups. Indeed, in the single-compartment SDR, each cells of the population are experiencing the same dissolved oxygen profile, whereas for the two-compartment SDR, cell-to-cell heterogeneity is higher since microbial cells are exposed to different dissolved oxygen profile according to the stochasticity of residence time in the respective compartments [35]. Comparison between the two SDR set-ups is thus very interesting for the evaluation of the extrinsic component of biological noise. The impact of scale-down conditions can be observed after 5 hours of culture (Figure 4). Indeed, higher acetate formation and lower growth rates are observed for the two SDR set-ups by comparison with the well-mixed reactor (Figure 4A and 4D). Accordingly, the RSG signal is also lowered in these SDR set-ups, indicating a drop of the metabolic activity (Figure 4C). An explanation at this level is the deviation of the electron flux from the oxidative phosphorylation pathway to the mixed acid fermentation pathway [36], leading to a decrease of the global electron flux through the electron transport chain. An interesting feature at this level is that the results are not significantly different between the two SDR set-ups (no significant differences observed at the level of acetate production, biomass yield and global RSG signal), suggesting that the mode of exposure of cells to dissolved oxygen, either stochastic or cyclic, has no strong influence. However, at this stage, only median RSG signal has been considered. As stated before, the interest of using flow cytometry relies on its ability to deliver information at the single cell level, giving access to the estimation of biological noise. In this context, cell to cell heterogeneity is becoming an important feature for optimizing bioprocess performances, since recent works report an important effect of phenotypic heterogeneity on metabolic activity of microbial cell [9][15]. However, the integration of phenotypic heterogeneity in the analysis of bioreactor performances is not a straightforward task [37]. For that purpose, we analyzed the mean to noise relationship of the RSG signal at the single cell level for the different cell populations collected under different cultivation conditions. Indeed, it has been previously shown that protein expression scale with noise, with a general correlation showing that noise is reduced when mean expression level increases. This correlation has been demonstrated for several

This article is protected by copyright. All rights reserved. 
microorganisms of industrial interest, such as E. coli [33] and Saccharomyces cerevisiae [38], and has also bee recently validated in bioprocessing conditions [39]. More recently, it has been proposed to use noise as an experimental parameter as an estimate of the consequences of phenotypic heterogeneity [40].

For that purpose, we applied the measurement of noise to cell population stained with RSG (Figure 5). In a first step, the results coming from the cultivation tests carried out with the E. coli strains expressing either VHb or ATPase have been processed in order to highlight the relationship between noise and mean RSG level (Figure 6A). As expected for the ATPase expressing strain, the mean RSG signal was lower and the value of the noise was higher. On the opposite, the VHb expressing strain exhibited high value of RSG intensity and a reduced amount of noise. The results coming from the scale-down experiments were further analyzed by using the same procedure (Figure 6B). The scale-down effect leads to a reduction of the mean RSG level, but no clear impact can be noticed at the level of the biological noise. Additionally, no differences can be observed between single or two-compartment SDR. Normally, a higher degree of noise would be expected for the two-compartment set-up considering the higher stochasticity at the level of the dissolved oxygen concentration profiles met at the single cell level in this specific set-up [41]. However, metabolite analysis point out that the sDo setups exhibit similar impact on acetate production (Figure $4 \mathrm{~A}$ ).

Concluding remarks

RSG can be used as an efficient metabolic biosensor related to the activity of the transport chain at the single cell level. This staining procedure is effective for the detection of carbon limitation and dissolved oxygen limitation at the single cell level.

Scale-down effect induced a decrease in the global RSG signal but had no significant effect of biological noise, which is surprising because an increase of the extrinsic component of noise under these fluctuating environmental conditions would have been expected. However, the global trend, i.e. reduced noise level when mean RSG activity is increased, has been observed for all the experiments. In the same context, no differences were observed between singlecompartment and two-compartment SDR, suggesting that the impact of stochasticity at the level of the circulation path followed by the individual cells cannot be tracked on the basis of RSG.

RSG can thus be used as a generic marker for the estimation of the metabolic activity of different E. coli strains in different operating conditions, but its use as a single cell marker for the detection of microbial phenotypic heterogeneity has to be considered with care and should be complemented with alternative analysis (e.g., by using more specific fluorescent protein based biomarkers).

Acknowledgements

This article is protected by copyright. All rights reserved. 
J.B., F.D., D.T. and A.D. gratefully acknowledge the Belgian fund for scientific research (FNRSFRS). JB and A.D. are supported by a PhD grant from FNRS-FRS (PDR project number 14730278). F.D. and A.R.L. acknowledge the bilateral grant FRS-FNRS-CONACyT number 209227. The authors would like to thank Tine Suhr for expert technical assistance during the construction of the ATPase expressing strain.

Conflict of interest statements

The authors have declared no conflict of interest

References

1. Enfors SO: Physiological responses to mixing in large scale bioreactors. J Biotechnol 2001, 85:175-185.

2. Neubauer P, Junne S: Scale-down simulators for metabolic analysis of large-scale bioprocesses. Curr Opin Biotechnol 2010, 21:114-21.

3. Delvigne F, Lejeune A, Destain J, Thonart P: Modelling of the substrate heterogeneities experienced by a limited microbial population in scale-down and in large-scale bioreactors. Chem Eng J 2006, 120:157-167.

4. Lapin A: Multi-Scale Spatio-Temporal Modeling: Lifelines of Microorganisms in Bioreactors and Tracking Molecules in Cells. Adv Biochem Eng Biotechnol 2010, 121:23-43.

5. Delvigne F, Goffin P: Microbial heterogeneity affects bioprocess robustness: Dynamic single cell analysis contribute to understanding microbial populations. Biotechnol J 2014, 9:61-72.

6. Swain PS, Elowitz MB, Siggia ED: Intrinsic and extrinsic contributions to stochasticity in gene expression. Proc Natl Acad Sci U S A 2002, 99:12795-12800.

7. Silva-Rocha R, de Lorenzo V: Noise and robustness in prokaryotic regulatory networks. Annu Rev Microbiol 2010, 64:257-275.

8. Shahrezaei V, Swain PS: The stochastic nature of biochemical networks. Curr Opin Biotechnol 2008, 19:369-374.

9. Delvigne F, Zune Q Lara AR, Al-Soud W, Sorensen SJ: Metabolic variability in bioprocessing: implications of microbial phenotypic heterogeneity. Trends Biotechnol 2014, 32:608-616.

This article is protected by copyright. All rights reserved. 
10. Boulineau S, Tostevin F, Kiviet DJ, ten Wolde PR, Nghe P, Tans SJ: Single-cell dynamics reveals sustained growth during diauxic shifts. PloS One 2013, 8.

11. Solopova A, van Gestel J, Weissing FJ, Bachmann H, Teusink B, Kok J, Kuipers OP: Bethedging during bacterial diauxic shift. Proc Natl Acad Sci U S A 2014, 111:7427-7432.

12. Van Heerden JH: Lost in Transition: Startup of Glycolysis Yields Subpopulations of Nongrowing Cells. Science 2014, 343:1-9.

13. Grunberger A, Wiechert W, Kohlheyer D: Single-cell microfluidics: opportunity for bioprocess development. Curr Opin Biotechnol 2014, 29.

14. Brognaux A, Han S, Sorensen SJ, Lebeau F, Thonart P, Delvigne F: A low-cost, multiplexable, automated flow cytometry procedure for the characterization of microbial stress dynamics in bioreactors. Microb Cell Factories 2013, 12.

15. Polizzi KM, Kontoravdi C: Genetically-encoded biosensors for monitoring cellular stress in bioprocessing. Curr Opin Biotechnol 2014, 31:50-56.

16. Zhang J, Jensen MK, Keasling JD: Development of biosensors and their application in metabolic engineering. Curr Opin Chem Biol 2015, 28.

17. Delvigne $F$, Ingels $S$, Boxus $M$, Thonart P: Bioreactor mixing efficiency modulates the activity of a prpoS::GFP reporter gene in E. coli. Microb Cell Factories 2009, 8:1475-2859.

18. Cabantous S, Terwilliger TC, Waldo GS: Protein tagging and detection with engineered selfassembling fragments of green fluorescent protein. Nat Biotechnol 2005, 23:102-107.

19. Nikolic N, Barner T, Ackermann M: Analysis of fluorescent reporters indicates heterogeneity in glucose uptake and utilization in clonal bacterial populations. BMC Microbiol 2013, 13.

20. Han S, Delvigne F, Brognaux A, Charbon GE, Sorensen SJ: Design of growth-dependent biosensors based on destabilized GFP for the detection of physiological behavior of Escherichia coli in heterogeneous bioreactors. Biotechnol Prog 2013, 29:553-563.

21. Hentschel E, Will C, Mustafi N, Burkovski A, Rehm N, Frunzke J: Destabilized eYFP variants for dynamic gene expression studies in Corynebacterium glutamicum. Microb Biotechnol 2013, 6:196-201.

22. Müller S: Origin and analysis of microbial population heterogeneity in bioprocesses. Curr Opin Biotechnol 2010, 21:100-113.

23. Konopka MC, Strovas TJ, Ojala DS, Chistoserdova L, Lidstrom ME, Kalyuzhnaya MG: Respiration response imaging for real-time detection of microbial function at the single-cell level. Appl Env Microbiol 2011, 77:67-72.

24. Sabido A, Martinez LM, de Anda R, Martinez A, Bolivar F, Gosset G: A novel plasmid vector designed for chromosomal gene integration and expression: use for developing a genetically stable Escherichia coli melanin production strain. Plasmid 2013, 69:16-23.

This article is protected by copyright. All rights reserved. 
25. Dikshit KL, Orii Y, Navani N, Patel S, Huang HY, Stark BC, Webster DA: Site-directed mutagenesis of bacterial hemoglobin: the role of glutamine (E7) in oxygen-binding in the distal heme pocket. Arch Biochem Biophys 1998, 349:161-166.

26. Koebmann BJ, Westerhoff HV, Snoep JL, Nilsson D, Jensen PR: The glycolytic flux in Escherichia coli is controlled by the demand for ATP. J Bacteriol 2002, 184:3909-3916.

27. Pablos TE, Mora EM, Le Borgne S, Ramirez OT, Gosset G, Lara AR: Vitreoscilla hemoglobin expression in engineered Escherichia coli: improved performance in high cell-density batch cultivations. Biotechnol J 2011, 6.

28. Pablos TE, Sigala JC, Le Borgne S, Lara AR: Aerobic expression of Vitreoscilla hemoglobin efficiently reduces overflow metabolism in Escherichia coli. Biotechnol J 2014, 9:791-799.

29. Lara AR: Living with heterogeneities in bioreactors - Understanding the effects of environmental gradients on cells. Mol Biotechnol 2006, 34:355-381.

30. Frey AD, Kallio PT: Bacterial hemoglobins and flavohemoglobins: versatile proteins and their impact on microbiology and biotechnology. FEMS Microbiol Rev 2003, 27:525-545.

31. Holm AK, Blank LM, Oldiges M, Schmid A, Solem C, Jensen PR, Vemuri GN: Metabolic and transcriptional response to cofactor perturbations in Escherichia coli. J Biol Chem 2010, 285:17498-17506.

32. Silander OK, Nikolic N, Zaslaver A, Bren A, Kikoin I, Alon U, Ackermann M: A genome-wide analysis of promoter-mediated phenotypic noise in Escherichia coli. PLoS Genet 2012, 8.

33. Taniguchi Y, Choi PJ, Li G-W, Chen H, Babu M, Hearn J, Emili A, Xie XS: Quantifying E. coli proteome and transcriptome with single-molecule sensitivity in single cells. Science 2010, 329:533-538.

34. Bar-Even A, Paulsson J, Maheshri N, Carmi M, O’Shea E, Pilpel Y, Barkai N: Noise in protein expression scales with natural protein abundance. Nat Genet 2006, 38:636-643.

35. Delvigne F, Destain J, Thonart P: Structured mixing model for stirred bioreactor : an extension to the stochastic approach. Chem Eng J 2005, 113:1-12.

36. Henkel SG, Ter Beek A, Steinsiek S, Stagge S, Bettenbrock K, de Mattos MJT, Sauter T, Sawodny O, Ederer M: Basic regulatory principles of Escherichia coli's electron transport chain for varying oxygen conditions. PloS One 2014, 9.

37. Delvigne F, Baert J, Gofflot S, Lejeune A, Telek S, Johanson T, Eliasson Lantz A: Dynamic single-cell analysis of Saccharomyces cerevisiae under process perturbation: comparison of different methods for monitoring the intensity of population heterogeneity. J Chem Technol Biotechnol 2015, 90:314-323.

This article is protected by copyright. All rights reserved. 
38. Newman JRS, Ghaemmaghami S, Ihmels J, Breslow DK, Noble M, DeRisi JL, Weissman JS: Single-cell proteomic analysis of S. cerevisiae reveals the architecture of biological noise. Nature 2006, 441:840-846.

39. Baert J, Kinet R, Brognaux A, Delepierre A, Telek S, Sorensen SJ, Riber L, Fickers P., Delvigne F: Phenotypic variability in bioprocessing conditions can be tracked on the basis of on-line flow cytometry and fits to a scaling law. Biotechnol J 2015, 10:1316-1325.

40. Liu J, Francois J-M, Capp J-P: Use of noise in gene expression as an experimental parameter to test phenotypic effects. Yeast Chichester Engl 2016.

41. Delvigne F, Destain J, Thonart P: A methodology for the design of scale-down bioreactors by the use of mixing and circulation stochastic models. Biochem Eng J 2006, 28:256-268.

This article is protected by copyright. All rights reserved. 
List of figures

Figure 1 : A evolution of the optical density (DO at $600 \mathrm{~nm}$ ) and the median of RSG signal (computed from a population of 40,000 cells analyzed by flow cytometry) during the cultivation of $E$. coli W3110 in batch bioreactor B evolution of the glucose and acetate concentrations

Figure 2 : effect of dissolved oxygen limitation on A the evolution of the median RSG signal of $E$. coli W3110 B the evolution of the median of the RSG signal for E. coli BL21 expressing VHb C evolution of the dissolved oxygen concentration during the experiment involving E. coli W3110 D evolution of the dissolved oxygen concentration during the experiment involving E. coli BL21 expressing VHb (aeration was stopped after 4.5 hours of culture)

Figure 3 : A evolution of the median RSG signal (computed from a population of 40,000 cells analyzed by flow cytometry) for E. coli K12 MG1655 expressing F1-ATPase in batch bioreactor B evolution of the glucose and acetate concentrations

Figure 4 : A evolution of glucose and B acetate concentration for the three bioreactor set-ups C evolution of the median RSG signal (computed from 40,000 microbial cells analyzed by flow cytometry) D evolution of the optical density at $600 \mathrm{~nm}$ for the three bioreactor set-ups

Figure 5 : distribution of RSG fluorescence among a population of 40,000 cells. Mean and standard deviation are exploited for estimating noise

Figure 6 : A relationship between mean and noise of the RSG signals for E. coli strains expressing respectively F1-ATPase and $\mathrm{VHb}$ in. The grey shaded area corresponds to the non fluorescent cells B relationship between mean and noise of the RSG signals corresponding to the cultivation of $E$. coli W3110 for the three operating conditions considered (i.e. well-mixed versus the two SDR conditions)

This article is protected by copyright. All rights reserved. 

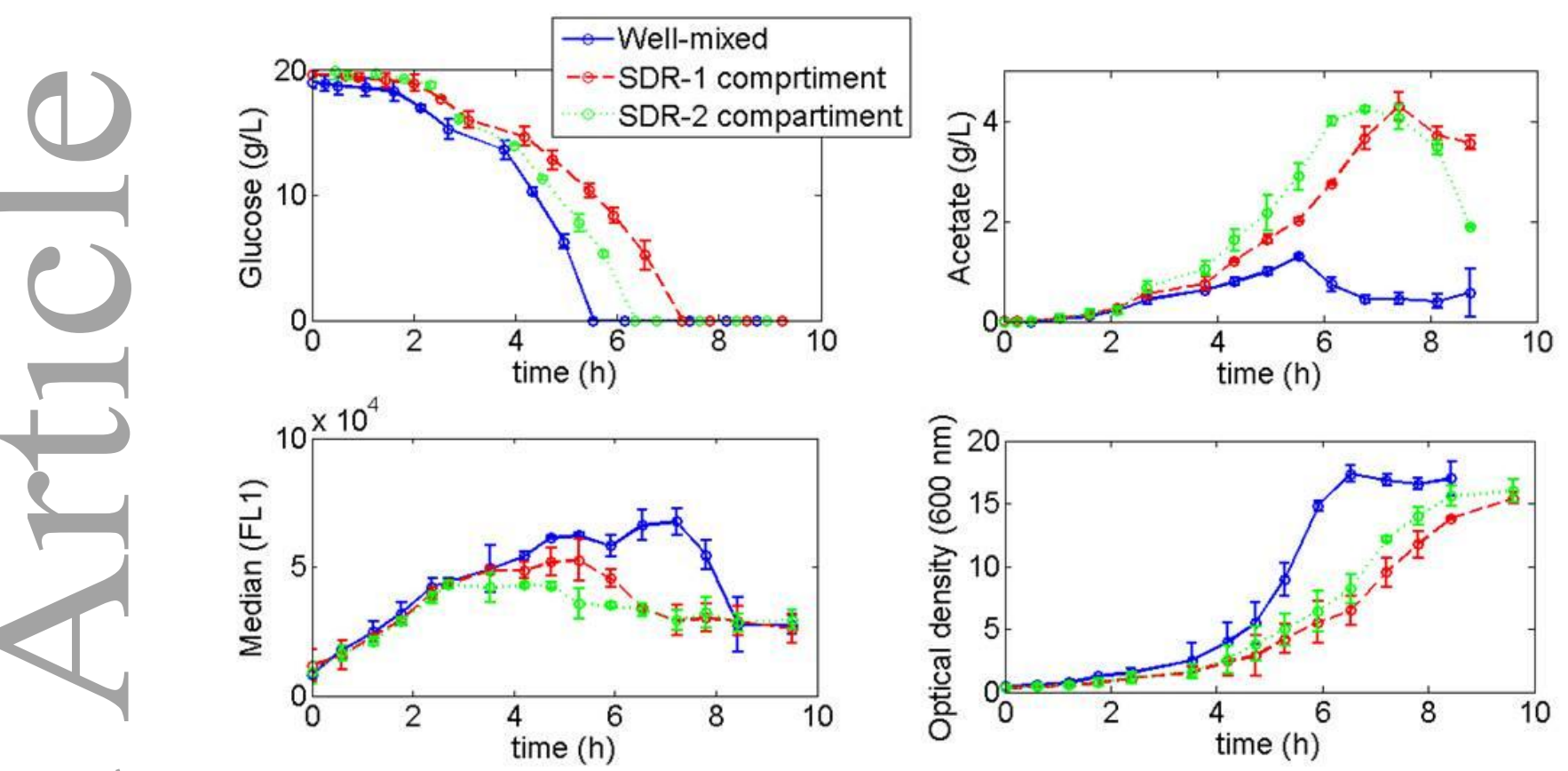

This article is protected by copyright. All rights reserved. 

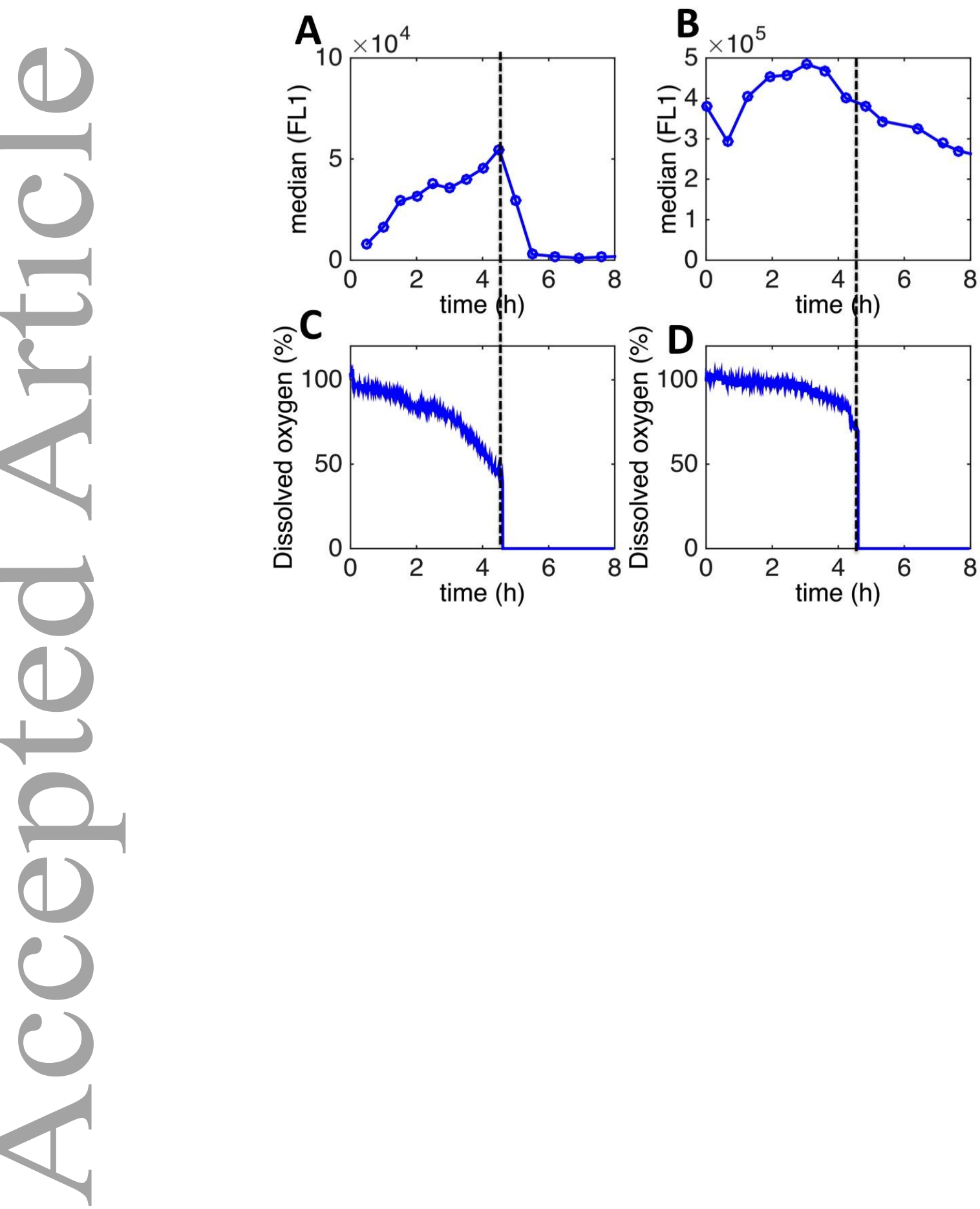

This article is protected by copyright. All rights reserved. 
A
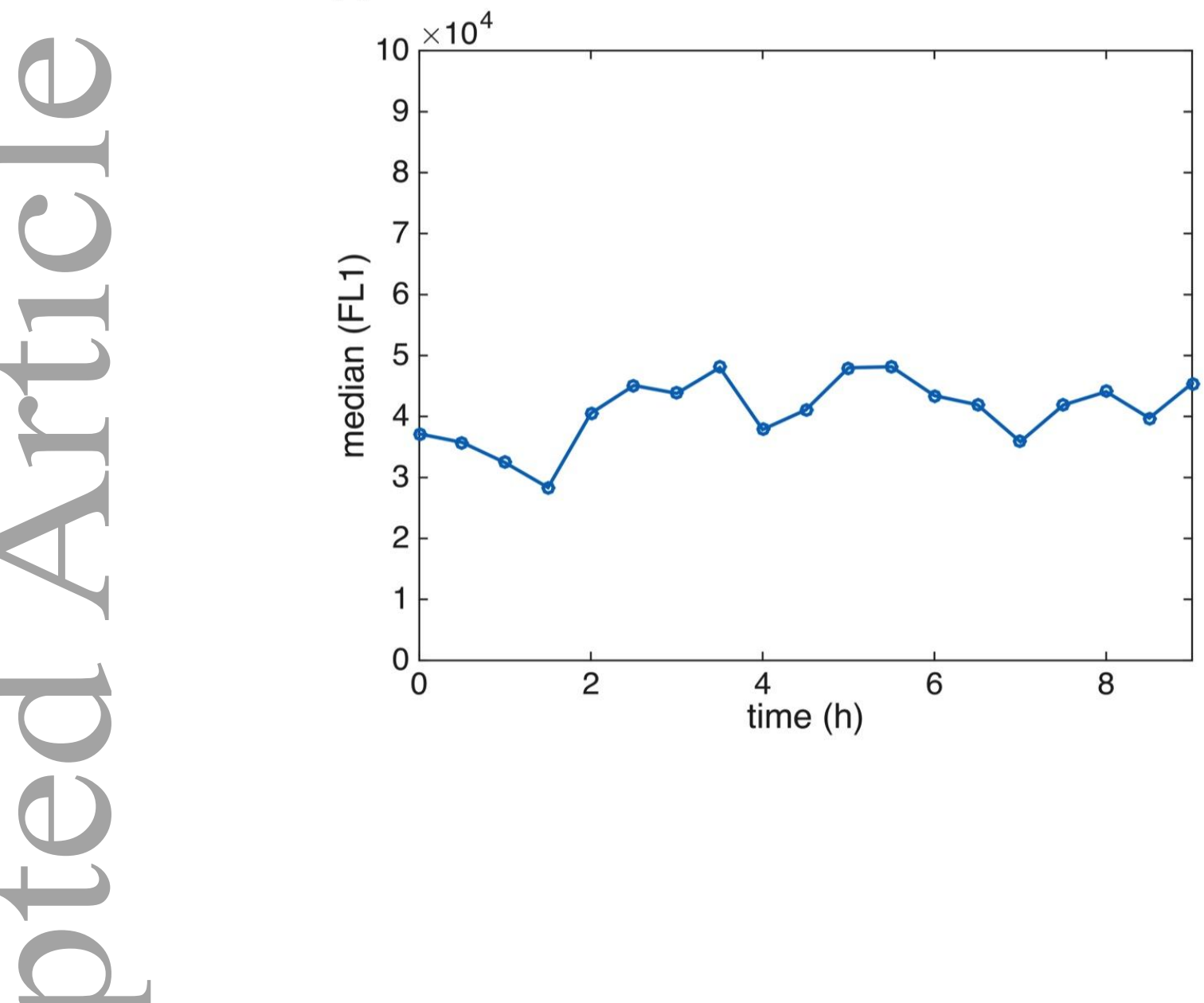

time $(\mathrm{h})$

This article is protected by copyright. All rights reserved. 

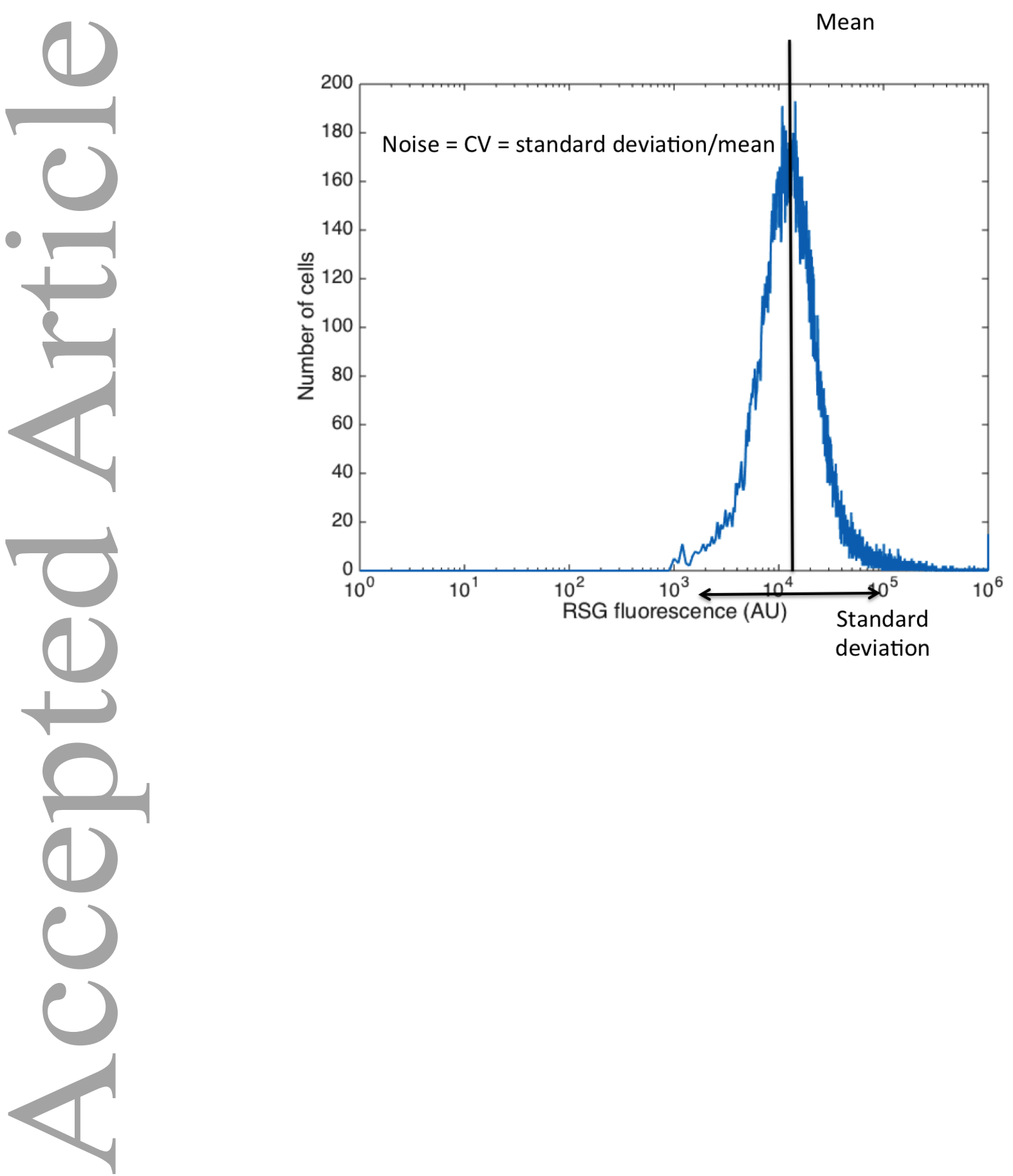

This article is protected by copyright. All rights reserved. 


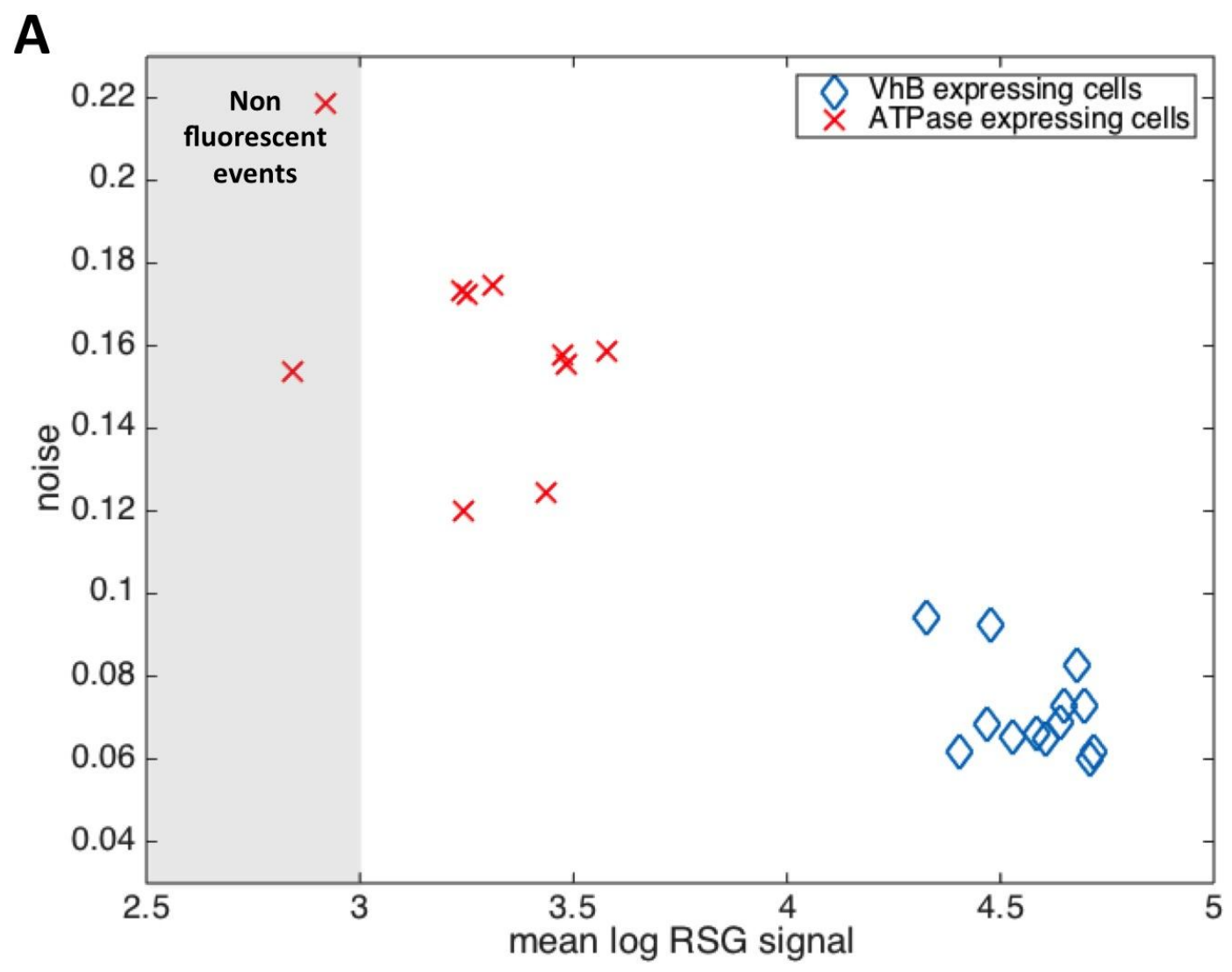

This article is protected by copyright. All rights reserved. 


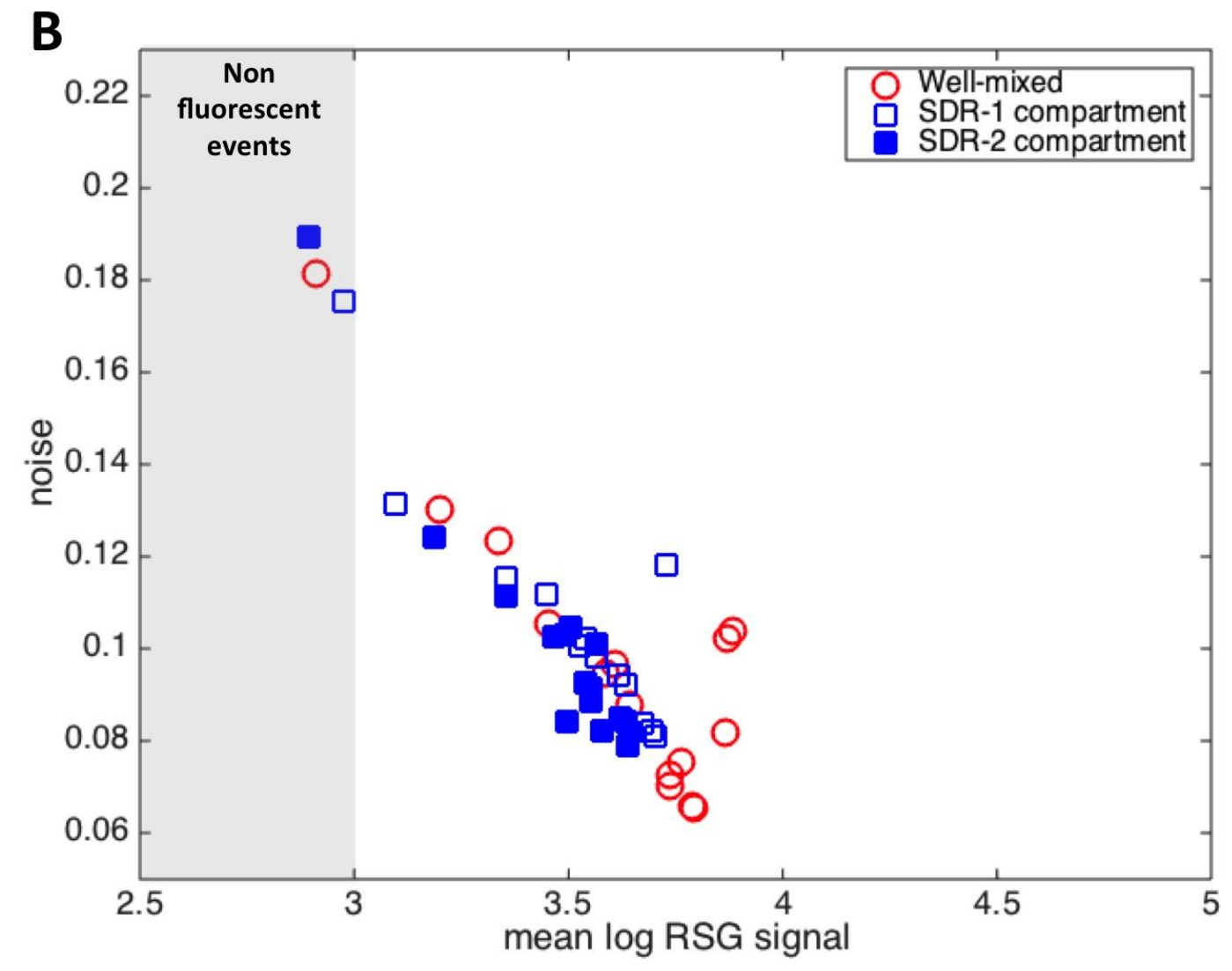

This article is protected by copyright. All rights reserved. 